\title{
Oral Surgery In Severe Thrombocytopaenia Patients: A Case-Control Comparison of Platelet Concentrate Versus Platelet Transfusion
}

\author{
Nadia Cocero' ${ }^{1}$, Laura Bergamasco², Marco Mozzati ${ }^{3}$ \\ ${ }^{1}$ Consultant, Oral Surgery Unit, Dentistry Section, Department of Surgical Sciences, University of Turin, Turin, ITALY. \\ ${ }^{2}$ Doctor, SIOM Oral Surgery and Implantology Center, Turin, ITALY. \\ ${ }^{3}$ Full Professor, Department of Surgical Sciences, University of Turin, Turin, ITALY.
}

\section{Key Words}

Thrombocytopaenia, platelet infusion, platelet rich in growth factors, PRGF

\section{Correspondence:}

Dr. Nadia Cocero,

Turin University Dental Faculty

Department of Oral Surgery

10100 Torino - Italy

e-mail: c_nadia77@libero.it

\begin{abstract}
Aim: Surgical treatment of patients with severe thrombocytopaenia $(<50,000$ platelets $/ \mu \mathrm{L})$ is a major problem for dentists due to an increased risk of bleeding. To reduce post-extraction haemorrhage, we tested a new approach using a platelet concentrate and compared its outcomes with those of routine platelet transfusion.

Methodology: Sixty-six patients with a platelet count between 29,000 and $46,000 / \mu \mathrm{L}$ were included in the study. Each patient was to undergo at least two sessions for the extraction of teeth; in one session the patients received a platelet transfusion before the extraction and in the other the post-alveolar sockets were treated with Plasma Rich in Growth Factors (PRGF). Patients were evaluated after $0.4,1,3$ and 7 days to assess bleeding and haematoma.

Results: Patients treated with PRGF consistently exhibited a statistically significant reduction in post-extraction bleeding, hematoma and need for re-intervention. There is ample evidence that application of PRGF in the post-alveolar socket markedly reduced the risk of haemorrhage and associated complications in all patients.

Conclusion: PRGF concentrate is recommended for the treatment of thrombocytopaenic patients due to its remarkable ability to stimulate healing while greatly reducing side effects, such as bleeding and haematoma.
\end{abstract}

(Int Dent Res 2012;2(2):33-36)

\section{Introduction}

The physiological range of circulating platelets (PLTS) lies between 150,000 and $400,000 / \mu \mathrm{L}$. A reduced number of platelets in peripheral blood, or thrombocytopaenia, is classified as mild $(75,000<$ PLTS $<150,000 / \mu \mathrm{L})$, moderate $(50,000<\mathrm{PLTS}<$ $75,000 / \mu \mathrm{L})$, or severe (PLTS $<50,000 / \mu \mathrm{L})(1)$.

The oral manifestations of thrombocytopaenia are petechiae and bruises in the areas of trauma (junction of the hard palate and soft palate, belly, tongue, cheek mucosa) and gingival bleeding. Perioperative complications consist of haemorrhage and bruising after surgery. An additional risk is represented by bacterial superinfection, which may cause postoperative bleeding due to lysis of the clot or septic complications (2).

For major oral surgery, the safety threshold is PLTS $>100,000 / \mu \mathrm{L}$. For PLTS $>50,000 / \mu \mathrm{L}$, simple procedures can be safely performed with local 
haemostasis. Patients with PLTS $<50,000 / \mu \mathrm{L}$ are routinely treated with a platelet transfusion; despite this precaution, bleeding and haematoma remain a concern $(3,4)$.

To overcome this problem, we tested an alternative approach using platelet concentrates: plasma rich in growth factors (PRGF).

The use of blood-derived products to seal wounds and stimulate healing was introduced by Kingsley (5), who used an autologous plateletderived preparation called platelet-rich plasma (PRP). Anitua et al. introduced a new protocol for platelet gel preparation, producing the so-called plasma rich in growth factors (PRGF) (6-9). PRGF does not use exogenous bovine thrombin as an activator, avoiding the risks of immunological reactions and disease transmission. These platelet gels are highly concentrated forms of autogenous platelets, known for their role in haemostasis; they prevent blood loss at sites of vascular injury by adhering, aggregating, and forming a pro-coagulant surface, leading to thrombin generation and fibrin formation. Platelets also release growth factors and active metabolites that promote tissue repair and influence the reactivity of vascular and other blood cells in angiogenesis and inflammation, so are useful in clinical situations requiring rapid healing and tissue regeneration $(10,11)$.

\section{Materials and Methods}

This study included 66 patients affected by severe thrombocytopaenia $(29,000<\mathrm{PLTS}<46,000 / \mu \mathrm{L})$ who required tooth extractions. The indications for tooth extraction included root or crown fractures, unrestorable caries, residual roots and periodontal and endodontic abnormalities. No exclusion criteria were applied regarding age, sex or the type of disease that led to thrombocytopaenia. This study conforms to the Helsinki Declaration of 1975 . Informed consent was obtained.

The baseline characteristics of our patients were: age $54.75 \pm 7.56$ years, males $73 \%$, average number of extractions $3.74 \pm 1.79$. Regarding concurrent diseases, $15 \%$ had diabetes, 9\% hypertension and heart diseases, none had chronic renal failure, and $3 \%$ had autoimmune diseases that required corticosteroid therapy.

Each patient required at least two sessions for single or multiple tooth extraction, allowing application of both methods, with each patient playing a double role as a case-control.

In the session named Protocol 0, the patient received a platelet transfusion, performed in a transfusion centre according to standard protocols, 30 min before the dental extraction. $(2,12,13)$.
In the session named Protocol 1 , prior to the extraction, $\sim 10-20 \mathrm{~mL}$ of blood was drawn into 5$\mathrm{mL}$ tubes containing $3.8 \%$ trisodium citrate solution as an anticoagulant. The tubes were centrifuged at $1800 \mathrm{rpm}$ for $8 \mathrm{~min}$ (PRGF SistemVR, BTI Biotechnology Institute, Milan, Italy) at room temperature. The blood was thus separated into its three basic components: red blood cells, at the bottom of the tube, PRGF in the middle, and plasma poor in growth factors (PPGF) at the top. The fraction $(0.5 \mathrm{~mL})$ located immediately above the erythrocytes was collected and transferred to sterile tubes. Calcium chloride $(50 \mu \mathrm{L})$ at $10 \%$ was added per $1 \mathrm{~mL}$ PRGF fraction. After 15 to $20 \mathrm{~min}$, the PRGF gel had formed (see Fig.1i) and was ready to be introduced into the post extraction socket. The time delay between PRGF gel formation and defect filling was 5-10 min. $(10,14)$.

All sessions were performed by the same surgeon who had extensive clinical expertise with both protocols, and were preceded by a platelet count: in the Protocol 0 session, the count was performed before and after the transfusion.

Following local anaesthesia (mepivacaine 3\% without adrenaline), the teeth were extracted in an atraumatic manner, with rotation and traction movements using dental forceps and elevator. The alveolar sockets were sutured with silk thread 3/0 and the suture was removed after 7 days. The same analgesic therapy (paracetamol $1000 \mathrm{mg}$, one tablet twice daily for 2 days) and postoperative instructions were given to all patients, regardless of the adopted protocol.

Bleeding and risk of haematoma were assessed at $0.4,1,3$ and 7 days following the extraction through an objective inspection of the oral cavity. In cases of bleeding that could not be stopped by gauze compression, the necrotic coat was removed and a new suture made.

The evolution of two clinical cases is illustrated in Fig.1. The first six panels refer to Protocol 0 and illustrate: preoperative XR (a), preoperative endoral vision (b), 4-teeth extraction (c), silk-thread suture (d), healing at the 3rd (e) and 7th day (f). The next six panels refer to Protocol 1: pre-operative XR (g), 6-teeth extraction (h), application of the PRGF inside the alveolar socket (i), silk-thread suture (I), healing at the 3rd (m) and 7th day $(n)$.

Continuous variables, relative to baseline and platelet data, were expressed as means \pm standard deviations and analysed using the paired t-test. Categorical data relative to the outcomes (bleeding, need for re-intervention and haematoma) of the two protocols were assembled in $2 \times 2$ tables. Null hypothesis $p$ (statistical significant $p<0.05$ ), risk ratios RR's for protocol 1 (the procedure under trial), and their $95 \% \mathrm{CIs}$ were estimated by the standard 
chi-square test with Yates correction or with the Fisher test. Used statistical programs: openepi.com and SPSS.
The main results are summarised in Table 1 . The percentages of the outcome parameters indicate the frequency of a given event in the 66 patients. Italics indicate statistical significance.

\section{Results}

TABLE 1: Comparison of the two protocols.

\begin{tabular}{|c|c|c|c|c|c|c|c|}
\hline & \multirow{2}{*}{$\begin{array}{l}\text { Platelet Count } \\
(/ \mu \mathrm{L})\end{array}$} & \multicolumn{4}{|c|}{ Bleeding } & \multirow{2}{*}{$\begin{array}{l}\text { Re-intervention } \\
\text { to stop bleeding }\end{array}$} & \multirow{2}{*}{ Hematoma } \\
\hline & & 0.4 days & 1 days & 3 days & 7 days & & \\
\hline Prot 0 & $\begin{array}{l}\text { Pre: } 38.02 \pm 4.33 \\
\text { Post: } 57.32 \pm 5.10\end{array}$ & $26 \%$ & $36 \%$ & $39 \%$ & $12 \%$ & $21 \%$ & $44 \%$ \\
\hline Prot 1 & $36.86 \pm 4.46$ & $5 \%$ & $9 \%$ & $9 \%$ & $6 \%$ & $0 \%$ & $14 \%$ \\
\hline p & 0.00008 & 0.002 & 0.0004 & 0.0001 & 0.36 & 0.03 & 0.0003 \\
\hline $\begin{array}{l}\text { RR } \\
(95 \% C)\end{array}$ & & $\begin{array}{c}0.01 \\
(0.1-0.6)\end{array}$ & $\begin{array}{c}0.25 \\
(0.1-0.6)\end{array}$ & $\begin{array}{c}0.23 \\
(0.1-0.5)\end{array}$ & $\begin{array}{c}0.50 \\
(0.2-1.6)\end{array}$ & 0.01 & $\begin{array}{c}0.31 \\
(0.2-0.6)\end{array}$ \\
\hline
\end{tabular}

The differences between the outcomes of the two protocols are striking. We have null hypothesis $p<0.05$ (indicating statistical significance) for the presence of bleeding at all checks except for the last one, when bleeding was also reduced in protocol 0 . The risk ratios were $<1$, and so were their $95 \% \mathrm{CI}$, documenting the protective role played by protocol 1. Similar considerations hold for the presence of haematoma and for the need for surgical reintervention. These results are remarkable considering that the platelet count at the protocol 1 session was significantly lower than that measured at the protocol 0 session before transfusion.

Some patients that required a large number of extractions had to undergo three surgical sessions. This allowed us to evaluate the persistence and reproducibility of the differences between the two protocols evidenced by the previous two sessions.

Post-extraction haemorrhage is expected to worsen with decreasing platelet numbers; to study the impact of the number of platelets on the outcome of the two protocols, the 66 patients were subdivided into two groups, using $35,000 / \mu \mathrm{L}$ as a watershed. For both protocols, the haemostatic effect was not significantly related to the platelet count. It should be noted that patients with $\leq 35,000 / \mu \mathrm{L}$ platelets treated with protocol 1 had a better outcome (average bleeding and haematoma incidence, 12 and 30\%) than patients with $>35,000 / \mu \mathrm{L}$ platelets treated with protocol 0 (average bleeding and haematoma incidence, 26 and $37 \%$ ).

The statistical significance of the differences between the two protocols was found to be independent of both the type of tooth and the maxillary or mandibular arch. In all cases $p<0.001$ with $\mathrm{RR} \sim 0,25$.
Finally, we analysed the influence of baseline characteristics on the outcome of one or both protocols. Neither age nor gender affected the occurrence of bleeding, re-intervention or haematoma. Among the concurrent diseases, only diabetes was associated with a statistically significant increase in risk. Diabetic patients treated with protocol 0 had a doubled risk of bleeding (from 25.9 to $50.0 \%$ ) and haematoma (from 37.0 to $90 \%$ ). Protocol 1 confirms its protective action for even this special class of patients by limiting the increase in risk from 7.1 to $12.5 \%$ for bleeding and 7.1 to $10.0 \%$ for haematoma.

\section{Discussion}

Our trial of the alternate use of platelet infusion and PRGF in a single patient suggested the latter to have superior outcomes in terms of delayed bleeding, haematoma and need for surgical reintervention. The results were statistically significant, reproducible over repeated sessions, and were unchanged in patients with platelets $\leq 35,000 / \mu \mathrm{L}$ or with diabetes.

The greater healing ability of protocol 1 with respect to protocol 0 can be ascribed to their different modes of action.

Protocol 0 aims to increase the circulating platelet level in order to reduce bleeding and haematomas; however, while the time required for healing and wound closure after a tooth extraction is $\sim 7$ days, the effect of the transfusion wears off in $\sim 2$ days, often leading to breakthrough bleeding on the third day following extractions. 
In contrast, protocol 1 acts directly on the site of bleeding, sealing the open wound with a high concentration of fibrin. The ability to alter the platelet concentration according to wound size allows uniform coverage of the entire wound and provides immediate haemostasis without fixation devices.

Furthermore, protocol 0 has the drawback of possible disease transmission associated with the platelet infusion, which increases with the number of surgeries needed in thrombocytopaenic patients. The platelet concentrate, on the other hand, is a heterologous substance that should minimize the risk of disease transmission and immunological reactions. The PRGF technique requires only $\mathrm{CaCl}_{2}$ to produce the platelet concentrate gel (15).

\section{Conclusion}

In conclusion, PRGF concentrate is recommended for the treatment of thrombocytopaenic patients due to its remarkable ability to stimulate healing while greatly reducing side effects, such as bleeding and haematoma.

\section{Acknowledgments}

The authors deny any conflicts of interest related to this study.

\section{References}

1. Afdhal N, McHutchison J, Brown R, et al. (2008) Thrombocytopaenia associated with chronic liver disease. J of Epatology 48: 1000-7.

2. Scully C, Cawson RA (2004) Medical problem in dentistry. Churchill Livingstone; 5th edition

3. Israel S, Schwetz N, Boyar R, et al. (2006) Bleeding disorders: characterization, dental considerations and management. J Can Dent Assoc 72(9):827

4. Mozzati M et al. (2008) Chirurgia Stomatologica Biologicamente Guidata. Approccio al paziente. Linee guida e protocolli operativi. UTET, Torino, pp 105-12.

5. Kingsley CS (1954) Blood coagulation; evidence of an antagonist to factor VI in platelet-rich human plasma. Nature 173:723-4.

6. Anitua E. (1999) Plasma rich in growth factors: Preliminary results of use in the preparation of future sites for implants. Int J Oral Maxillofac Implants 14:529-35.

7. Anitua E, Andia I, Ardanza B, et al. (2004) Autologous platelets as a source of proteins for healing and tissue regeneration. Thromb Haemost 91:4-15.

8. Anitua E, Sanchez M, Nurden AT, et al. (2006) New insights into and novel applications for platelet-rich fibrin therapies. Trends Biotechnol 24:227-34.

9. Anitua E, Sanchez M, Orive G, Andia I (2007) The potential impact of the preparation rich in growth factors (PRGF) in different medical fields. Biomaterials 28:4551-60.

10. Mozzati M, Martinasso G, Pol R et al. (2010) The impact of plasma rich in growth factors on clinical and biological factors involved in healing processes after third molar extraction. Journal of Biomedical Materials Research 741-6.

11. Mazzucco L, Medici D, Serra M et al. (2004) The use of autologous platelet gel to treat difficult-toheal wounds: a pilot study. Transfusion 1013-8.

12. Chiarini L, Bertoldi C, Narni F (1997) Management of patients with coagulation disorder in oral and maxillofacial surgery. Management of patients with hypocoagulation caused by primary thrombocytopathy. Minerva Stomatol 46(1-2):61-71.

13. Gortzak RA, Huijgens PC (1999) A patient with thrombocytopaenia: what do I do? Ned Tijdschr Tandheelkd 106(12):459-60.

14. Mozzati M et al. (2008) Chirurgia Stomatologica Biologicamente Guidata. Complementi per la chirurgia e la biostimolazione. UTET, Torino, pp 117-60.

15. Landesberg R, Moses M, Karpatkin M (1998) Risk of using Platelet Rich Plasma Gel. J Oral Maxillofac Surg 56:1116-7. 